\title{
Pedigree investigation of severe hemolytic disease of the newborn caused by rare anti-Jk3
}

\author{
Zhiyuan $\mathrm{Xu}^{1, \triangle}$, Yushiang $\mathrm{Lin}^{2, \triangle}$, Haochun Chang ${ }^{2}$, Xiaofei $\mathrm{Li}^{1}$, Daowong Fan ${ }^{1}$, Yan Qiu ${ }^{1}$, \\ Guangyan Zhuang ${ }^{1}$, Tianhong Miao ${ }^{1, *}$ \\ 'Blood Group Lab, Beijing Red Cross Blood Center, Beijing 100191, China; \\ ${ }^{2}$ Department of Clinical Medicine, School of Basic Medical Sciences, Peking University Health Science Center, Beijing 100191, China
}

\begin{abstract}
This study was designed to evaluate the molecular and serological features of a newborn with severe hemolytic disease of the newborn (HDN) caused by anti-Jk3, and to further richour understanding of Kidd subgroup genetics using pedigree analysis, which is the first analysis of a Jk null phenotype in China and the first report of severe HDN caused by anti-Jk3. A female baby presented with hyperbilirubinemia $(36 \mu \mathrm{mol} / \mathrm{L})$ on the day of birth. Antibody screening tests using blood samples from the patient and her family indicated that the mother's plasma contained alloantibodies against high frequency antigens, and the results of direct Coombs test were all negative. Kidd phenotypes were $\operatorname{Jk}(\mathrm{a}-\mathrm{b}-), \operatorname{Jk}(\mathrm{a}-\mathrm{b}+)$, and $\mathrm{Jk}(\mathrm{a}-\mathrm{b}+)$ in the mother, father, and baby, respectively. Kidd genotype was determined by PCR amplification of a single nucleotide polymorphism (838) and all family members were $\mathrm{Jk}(\mathrm{a}-\mathrm{b}+)$. Kidd gene exons 4 to 11 were sequenced to identify potential mutations. Sequencing analysis revealed that c.838 $\mathrm{G}>\mathrm{A}$ and intron c. $3-78 \mathrm{G}>\mathrm{A}$ homozygosity occurred in all family members along with homozygosity and heterozygosity for c.IVS5-1G $>$ A in the mother and newborn, respectively. In conclusion, serological and genetic analyses confirmed that the $\mathrm{Jk}(\mathrm{a}-\mathrm{b}-)$ phenotype was caused by homozygous IVS5-1G $>\mathrm{A}$ mutation of the Kidd gene. This result is consistent with that of a previous report and presents a useful diagnostic tool to identify HDN caused by anti-Jk3. A further study is required to identify the effect of intron $3-78 \mathrm{G}>\mathrm{A}$ mutation on phenotype.
\end{abstract}

Keywords: Jk(a-b-), anti-Jk3, Hemolytic disease of newborn, IVS5-1G>A

\section{INTRODUCTION}

The SLC14A1 locus is located on chromosome 18 at $18 \mathrm{q} 11-\mathrm{q} 12$, spans approximately $30 \mathrm{~kb}$ containing 11 exons, and constitutes the Kidd (Jk) blood group system $\left(\right.$ ISBT009) ${ }^{[1]}$. The Kidd glycoprotein is a transmembrane protein and functions as a urea transporter. Kidd

*Correspondence to: Tianhong Miao, Director of Blood Group Lab, Beijing Red Cross Blood Center, Beijing 100191, China; Tel: 008615201107318, E-mail: tianhongm@163.com.

The authors have no conflicts of interest to declare. antigens $\mathrm{Jka}$ and $\mathrm{Jkb}$ represent a polymorphism and are produced by alleles with Asp280 and Asn280 substitutions, respectively. Jk $(\mathrm{a}-\mathrm{b}-)$ is a rare null phenotype lacking the high incidence Jk3 antigen. Jk (a-b-) red blood cells are easily identified due to their failure to lyse in $2 \mathrm{M}$ urea, distinguishing them from other Kidd phenotypes with normal urea transporter function ${ }^{[1]}$.

Natural or transfused immune anti-Jk3 has been identified in people with $\mathrm{Jk}(\mathrm{a}-\mathrm{b}-)$, and anti-Jk3 is responsible for severe immediate and delayed hemolytic transfusion reactions. However, the severe clinical problems of babies born to mothers with anti-Jk3 have not yet displayed with a positive direct Coombs 
test $^{[2,3]}$.

The aim of this study was to conduct a pedigree investigation of a baby with HDN through serological and molecular analysis of the Kidd protein and SLC14Al (Kidd) gene. Notably, this is the first study to demonstrate HDN caused by rare anti-Jk3 in Asia.

\section{MATERIALS AND METHODS}

\section{Brief HDN case report}

A live female baby, born at term to a 35-year-old mother (gravida 2, para 1) without transfusion records, was admitted to the neonatal unit with hyperbilirubinemia $(28 \mu \mathrm{mol} / \mathrm{L})$, which elevated to $(36 \mu \mathrm{mol} /$ L) after 24 hours. The laboratory investigations concerning the baby were as follows: peripheral blood revealed anemia, the direct Coombs test (DAT) with monoclonal anti-IgG was positive, and DAT with anti-IgM was negative. The baby's blood group was $\mathrm{B}$, ccDEE, and Jk (a-b+). The mother's blood group was $\mathrm{O}$, ccDEE, and Jk (a-b-) and the father's blood group was B, CcDEe, and Jk (a-b+) (Figure 1). Antibody screening tests and identification of alloantibodies against high prevalence antigens with DAT were negative in the mother's plasma. This is known to react with Jk $(a+b+)$ cell as 1:32 titers. The baby's plasma reacted as $3+$ titers in the gel agglutinin test with all panel and screen cells. The baby was treated with a double volume exchange transfusion from $\mathrm{O}+$ blood donor immunoglobulins and phototherapy.

\section{Blood samples and DNA extraction}

Peripheral EDTA-treated anti-coagulated blood was stored at $4{ }^{\circ} \mathrm{C}$. Serological tests and DNA extraction were performed within 12 hours of blood acquisition. DNA was purified from the buffy coat using micromagnetic technology from a commercial kit (Prepito DNA Blood 250 Kit, Chemagen, Germany) and an automatic instrument (ChemagicPrepito, Chemagen, Germany). All DNA samples were stored at $-80^{\circ} \mathrm{C}$ until required for molecular analysis.

\section{Antibody screening, identification, and phe- notype analysis}

The screen and identification panel cells used at the Blood Group Laboratory (BGL) of the Beijing Red Cross Blood Center (BRCBC) cover a vast proportion of antigen systems within Asia. These antigen systems include Rh, MNS, Duffy, Kidd, Kell, Lewis, P1, $\mathrm{Xg}$, and Lutheran, and low-frequency antigens such as Dia and Mur (Bio-Rad Laboratories GmbH, Germany, and Jiangsu Libo Medicine Biotechnology co., Ltd, China). Detection methods included conventional tube techniques performed at room temperature and gel column tests (Bio-Rad Laboratories GmbH, Germany and Jiangsu LiBio Biotech, China). The aberrant phenotype was confirmed using the column agglutination test (Bio-Rad Laboratories $\mathrm{GmbH}$ Munchen, Germany, and Jiangsu LiBio Biotech, China) and tube technique (Sanquin GmbH, Netherland). All other procedures, such as phenotyping, sample reception, report documentation, and report writing were based on the regulations and standard procedures specified by the BGL of the BRCBC.

\section{Genotyping and molecular analysis}

Kidd blood group genotyping was performed by polymerase chain reaction (PCR) with sequencespecific primers using a commercial kit (multierythrocyte antigengenotype, Jiangsu LiBio Medicine Biotechnology, China). Sanger sequencing of Kidd exons 4 to 11 was performed using a commercial Kidd gene sequencing kit (Kidd E4-11 sequence, Jiangsu LiBioMedicine Biotechnology, China). Sequencing of PCR purified products was performed by Sangon Biotech (Beijing, China) and the results were analyzed using sequence analysis software (Geneious R9, New Zealand). The SLC14AI allele sequence (GenBank No. NM 015865.1) template was used as a reference for analysis and to mark the mutations.

\section{RESULTS}

\section{Jka and Jkb phenotyping and genotyping}

Kidd phenotyping using the tube technique showed phenotypes of $\mathrm{Jk}(\mathrm{a}-\mathrm{b}+)$ in the baby and her father, and Jk (a-b-) in the baby's mother (Fig. 1). In contrast, genotyping of SLCI4AI exon 9 (nt. 838) by $\mathrm{PCR}$, with primers designed to identify the single Jka/ $\mathrm{Jkb}$ nucleotide polymorphism, revealed that all family members were Jk (a-b+) (Fig. 2). Considering the difference between genotypings, phenotyping of $\mathrm{Jk}$ null ruled out deletion mutations at SNPs.

\section{Sequence of SLC14A1 exons 4 to 11}

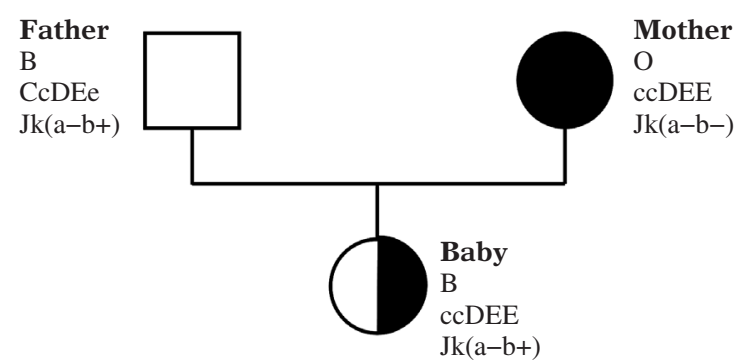

Fig. 1. Pedigree of the baby and her family based on ABO, Rh, and Kidd phenotypes. $\mathrm{ABO}, \mathrm{Rh}$, and Kidd phenotypes are indicated as the top, middle, and bottom designation, respectively, for each family member. 


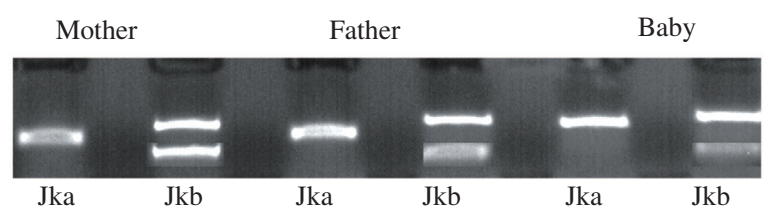

Fig. 2 Kidd genotyping. The upper band represents the internal control and the lower band represents a positive allele.

Kidd sequencing results demonstrated that all three family members were homozygous at the Jka/JkbSNP c.838 G>A. The mother was identified as homozygous for c.IVS5-1G>A, causing Jk null expression, and the newborn was heterozygous for this mutation. All three family members were homozygous for a mutation identified in intron 3 (nt. $78 \mathrm{G}>\mathrm{A}$ ) (Fig. 3). The mutation of c.IVS5-1G $>$ A plays an important role in RNA splicing, and results in the loss of exon 6 from mRNA transcripts. The glycoproteins produced by the Kidd gene form the urea transporter. In the absence of functional urea transport, the mother's erythrocytes remained intact in a $2 \mathrm{M}$ urea solution.

\section{Discussion}

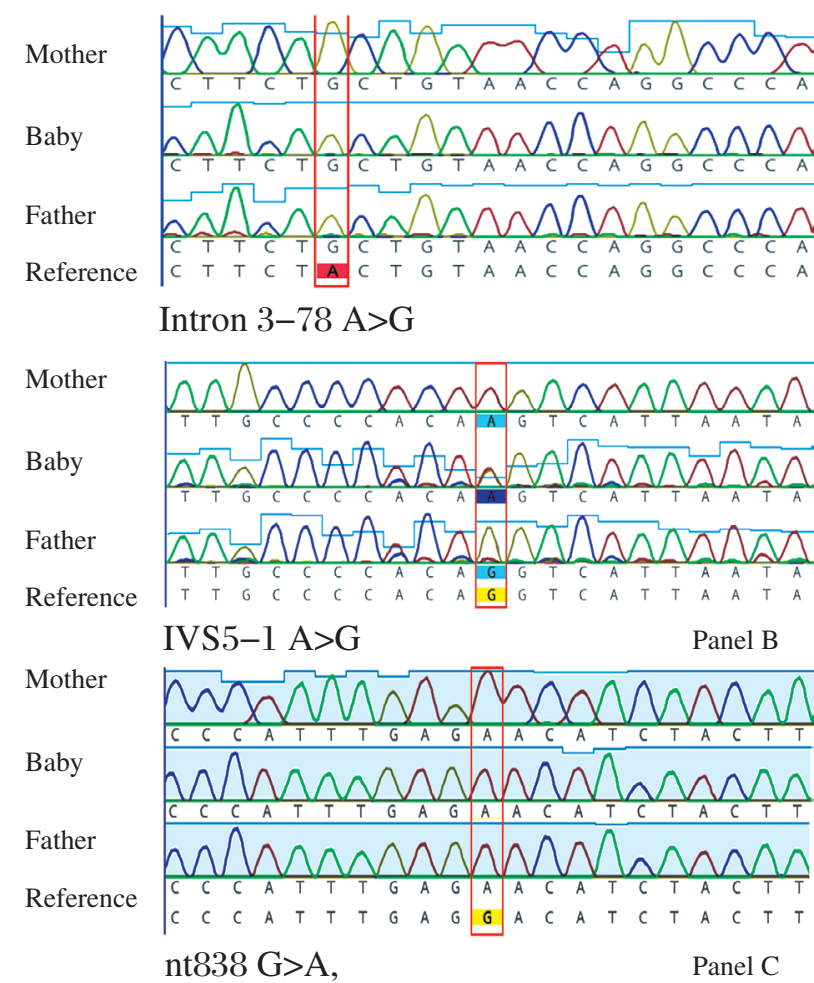

Fig. 3. Pedigree of the baby and her family according to the probable Kidd exon 4 to 11 sequence. Panel A shows a part of the results from intron 3 , the square indicates the -78 homozygous SNP in the family. Panel B shows a part of the intron 5 sequencing results, the square indicates the -1 homozygous and heterozygous SNP in the mother and baby as the key SNP in Jk null. Panel C shows a part of the exon 9 sequencing results, the square indicates the SNP at nt. 838 , homozygous in all family members as the key SNP in Jka/Jkb.
The Jk null phenotype is very rare in all ethnic populations and is naturally, or secondarily, immune to anti-Jk3, which causes hemolytic reactions. The highest frequency of $\mathrm{Jk}(\mathrm{a}-\mathrm{b}-)$ occurs in Polynesians at $0.27 \%$. In other populations, the Jk $(a-b-)$ frequency has been measured at $0.002 \%$ (Japanese), $0.003 \%$ (Finnish), and $0.02 \%$ (Thai) ${ }^{[4]}$. Epidemical studies show that the frequency of the Jk null phenotype in southeastern China (Shenzhen) is $0.019 \%$ (out of 20453 blood donors) ${ }^{[5]}$, central China (Henan) is $0.03 \%$ (6391 blood donors) ${ }^{[6]}$, eastern China (Shanghai) is $0.0041 \%$ (24200 blood donors $)^{[7]}$, southwestern China (Chengdu) is $0.02 \%$ (29498 blood donors $)^{[8]}$, northern China is $0.0044 \%$ (Huhehot) (22309 blood donors ${ }^{[9]}$, and in western China the Jk null phenotype has never been observed (Xinjiang; in 1062 blood donors ${ }^{[10]}$. There are only two published articles reporting anti-Jk3 in China. Lo K et al. ${ }^{[11]}$ identified eight Jk null donors in 32000 Guangzhou blood donors, and antibody screen tests did not reveal anti-Jk3. Zhen $\mathrm{X}^{[12]}$ reported one pregnant women with anti-Jk3 in Guangzhou through routine prenatal examination and enlisted three Jk null donors to provide blood to support her through the remainder of pregnancy. Our research searching into the PubMed database revealed eight articles that discussed anti-Jk3. Two articles reported investigations of severe pre-transfusion anemia $^{[13,14]}$. Three articles were relate to anemia in pregnant women, including severe hemolytic reaction due to anti-Jk3, and all three articles reported that the newborns remained unaffected ${ }^{[15-17]}$. To date, only mild HDN due to anti-Jk3 has been reported in newborns ${ }^{[18-20]}$. Our study is the first one to report HDN in the Chinese and to document severe hemolytic reactions in HDN.

In all, twenty-eight mutations causing Jk null phenotypes have been reported in the literature. These mutations include eight single nucleotide mutations and encompass three SNPs, which occur in introns 1, 7 , and 5 . Two of these mutations form nucleotide deletions over $1.6 \mathrm{~kb}$ in length spanning exons 4 and 5 , and one SNP deletion occurs at nt. 327. The remaining 20 mutations caused Jk null phenotypes due to SNPs resulting in amino acid changes. The c.IVS5-1G>A mutation leads to an RNA splicing site error resulting in a loss of exon 6 from mRNA transcripts, and it is the major molecular etiology of Jk null phenotypes in China ${ }^{[21-23]}$. These results were supported by the pedigree investigation reported here (Figure 2, panel B). Interestingly, the SNP of intron 3 (at nt. 78) was first published in a Chinese Jk null report ${ }^{[24]}$. All family members described here, including the father and the baby with the normal $\mathrm{Jk}(\mathrm{a}-\mathrm{b}+)$ phenotypes were 
homozygous for the $\mathrm{G}>\mathrm{A}$ change. It is suggested that the intron 3 c. $78 \mathrm{G}>\mathrm{A}$ is a silent mutation in Chinese people. These results show that further investigation of Kidd gene sequences in China is required.

Our study shows that anti-Jk3 causes severe HDN and it is necessary to sequence the Kidd gene to identify and confirm the Jk null mechanism.

\section{References}

[1] Geoff Daniels. Kidd Blood Group Systems.In:Geoff Daniels Editor.Human blood groups, 3rd edition. Bristol, UK, 2013:325-6.

[2] Sands JM, Gargus JJ, Fröhlich O, et al. Urinary concentrating ability in patients with $\mathrm{Jk}(\mathrm{a}-\mathrm{b}-)$ blood type who lack carrier-mediated urea transport. J Am SocNephrol, 1992; 2:1689-96.

[3] Issitt PD, Obarski G, Hartnett PL, et al. Temporary suppression of Kidd system antigen expression accompanied by transient production of anti-Jk3. Transfusion, 1990; 30:46-50.

[4] Geoff Daniels. Kidd Blood Group Systems.In:Geoff Daniels Editor.Human blood groups, 3rd edition. Bristol, UK, 2013:329.

[5] Zheng W, Li D, Wang H, et al.Genomic characterization of the Jk( $\mathrm{a}-\mathrm{b}-)$ phenotype for children in Shenzhen, China. Chinese Journal of Blood Transfusion (in Chinese), 2015; 28(1): 24-5.

[6] Hao L, Du Z and Jin Z. Two cases report of jk(a-b-) and distributions in variety of different races. Acta AnthropologicaSinica(in Chinese), 1991; 10(3): 206-10.

[7] Zhu Z, Sheng W, Cheng H, et al. Rare blood group screening among Chinese blood donors in Shanghai area. Chinese Journal of Blood Transfusion (in Chinese), 2012; 15(4): 232-3.

[8] Hung Y, Xu Y, Wang H, et al. The frequency of Jk(ab-) phenotype in Chengdu blood donors. Chinese Journal of Blood Transfusion (in Chinese), 2011; 24(4): 333.

[9] Shang J, Jia W and Su R. The frequency of Jk(a-b-) phenotype in Huhehot blood donors. Chinese Journal of Blood Transfusion (in Chinese). 2011, 27( $7^{\text {th }}$ CSBT, abstract):6.

[10] Chiu F, Tian S, Baja T, et al. The frequency of Kidd phenotype in Uygur, Hui population at Xinjiang area. Chinese Journal of Blood Transfusion (in Chinese), 2012; 25(7): 682-3.
[11] Lo K, Wong T, Xia W, et al. Discovery to anti-Jk3 and pedigree investigation of Jk null. Chinese Journal of Blood Transfusion (in Chinese), 2008; 21(3): 172-3.

[12] Zheng X. Rare anti-Jk3 in Guangzhou.Herald Medicine (in Chinese), 2007, A02:1.

[13] McCaskill S, Wise S and Tinsley S. Anti-Jk3 in a Filipino man. Immunohematology, 2015; 31(3):119-22.

[14] Yousuf R, Abdul Aziz S, Yusof N, et al. A rare case of anti-jk3 antibody detected on pre-transfusion investigation. Indian J Hematol Blood Transfus, 2014; 30(3):208-10.

[15] Kuczmarski CA, Bergren MO and Perkins HA. Mild hemolytic disease of the newborn due to anti-Jk3: a serologic study of the family's Kidd antigens. Vox Sang, 1982;43(6):340-4.

[16] Ellisor SS, Reid ME, O’Day T, et al. Autoantibodies mimicking anti-Jkb plus anti-Jk3 associated with autoimmune hemolytic anemia in a primipara who delivered an unaffected infant. Vox Sang, 1983;45(1):53-9.

[17] Marshall CS1, Dwyre D, Eckert R, et al. Severe hemolytic reaction due to anti-JK3. Arch Pathol Lab Med, 1999; 123(10):949-51.

[18] Jator EK. Notorious anti-Jk3 in a pregnant woman. Clin Lab Sci, 2014; 27(2):78-82.

[19] Goldman H, Levinsky D. Hemolytic disease of the newborn caused by anti-JK3. Obstet Gynecol, 1982; 60(4):526-8.

[20] Pierce SR, Hardman JT, Steele S, et al. Hemolytic disease of the newborn associated with anti-Jk3. Transfusion, 1980; 20(2):189-91.

[21] Liu HM, Lin JS, Chen PS ,et al. Two novel Jk(null) alleles derived from $222 \mathrm{C}>\mathrm{A}$ in Exon 5 and $896 \mathrm{G}>\mathrm{A}$ in Exon 9 of the JK gene. Transfusion, 2009; 49(2):25964.

[22] Guo Z, Wang C, Yan K, et al. The mutation spectrum of the JK-null phenotype in the Chinese population. Transfusion, 2013; 53(3):545-53.

[23] Ma L, Liu YC, Zhu SW, et al. A novel missense mutation nt737T $>\mathrm{G}$ of $\mathrm{JK}$ gene with $\mathrm{Jk}(\mathrm{a}-\mathrm{b}-)$ phenotype in Chinese blood donors. Transfus Med, 2015; 25(1):38-41.

[24] Zhu F, Xu X, Hong X, et al. Research on molecular genetic basis for Jk(a-b-)phenotype. Chinese Journal of Blood Transfusion (in Chinese), 203; 16(4): 245-7.

(Received 02 March 2017, Revised 09 March 2017, Accepted 12 March 2017) 